\section{Neue Steuerung für Galvanikanlagen}

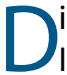
e Anlagensteuerung Galvatec von Icom Automation ist eine Automatisierungslösung mit komfortabler Bedienung und übersichtlicher Visualisierung. Sie zeichnet sich nach eigenen Angaben durch Flexibilität, hohe Ausfallsicherheit und ein ausgereiftes Protokollierungsystem aus. Neben einfacher intuitiver Artikelprogrammierung bieten die Steuerung Funktionen wie frei programmierbare Badbehandlungs- und Toleranzzeiten oder automa- tische Stromdichtenkorrektur innerhalb vorgegebener Grenzen. Das Programmsystem Lava.SQL stellt eine Vielzahl von Funktionen zur Datenverarbeitung im Umfeld des Galvanikbetriebs bereit. Neben der Hauptaufgabe der Erfassung, Überprüfung und Leitung der Badversorgung und -pflege, erlaubt es die Verwaltung und Protokollierung der laufenden Anlagenwartung.

Tel. 03677 84880, www.icom-automation.de

Halle 3, Stand D 54(16)

\title{
Filter für aggressive Medien
}

$M$ it einer umfassenden Palette an Filtern für aggressive Flüssigkeiten präsentiert sich Siebec auf dem ZVOGemeinschaftsstand. Im Spritzgussverfahren aus Kunststoff (PP/PVDF) hergestellt, sind die Filtertöpfe beständig gegenüber Säuren und Laugen. Die für die jeweiligen korrosiven Mittel geeigneten Pumpen haben eine Förderleistung von
0,2 bis 38 Kubikmeter pro Stunde und eine maximale Gesamtförderhöhe von 45 Metern. Die Pumpenkörper aus Spritzgusskunststoff (PP oder PVDF) weisen nach Herstellerangaben eine hervorragende Beständigkeit gegenüber Säuren und Laugen bis zu $110^{\circ} \mathrm{C}$ auf.

Tel. +33 4762612 09, www.siebec.com

Halle 3, Stand D54(24)

\section{Reinigung und Vorbehandlungsanlagen}

$D_{k}^{e-1}$ er Reinigung und Vorbehandlung kommt in der Oberflächentechnik eine immer stärkere Bedeutung zu. Branchenunabhängig steigt die Nachfrage nach automatischen oder teilautomatischen Reinigungs- und Strahlanlagen. Um diesem Trend Rechnung zu tragen, hat Heimer Lackieranlagen \& Industrielufttechnik in weitere Fertigungszentren investiert und stellt die Vorbehandlung in den Mittelpunkt der Präsentation auf der Hannover Messe. Hier kann der Besucher eine Reinigungssektion für hohe Fertigungsqualität besichtigen. Die ausgestellte Anlage ist nur eine von vielen möglichen Varianten, die auf den jeweiligen Bedarf abgestimmt werden. So umfasst das Angebotsspektrum sowohl Mehrzonen-Kammeranlagen mit automatischen Schiebetürsystemen, als auch Mehrzonen-Durchlaufanlagen einschließlich der Steuerungstechnik.

Tel. 05205 9813-0, www.heimer.de

Halle 3, Stand D 02

\section{Verbesserte Feuerverzinkungsprozesse}

Eontaine Technologie stellt auf der - Hannover Messe verbesserte Feuerverzinkungsprozesse nach ISO 1461 samt einer Auswahl an binären und ternären Legierungen vor. Die ZinqTechnologie ist das erste Single-DipFeuerverzinkungsverfahren, das die Verwendung von Zinkschmelzen mit mehr als 1\% Aluminiumgehalt sowie mit Zulegierung von Magnesium er- möglicht. Die Thermodiffusionstechnologie unter Einsatz von binären und ternären Zinklegierungen ermöglicht die Erschließung neuer Anwendungen für das Stückverzinken und kombiniert eine gesteigerte Ressourceneffizienz mit einem höheren Korrosionsschutz.

Tel. 0209 319270-0, www.fontaine-technologie.net Halle 3, Stand D 54(29)
Seit über 25 Jahren die passende Lösung zum effektiven Aufhängen und Abdecken bei der industriellen Lackierung. Riesiges Lager und Sonderentwicklungen komplett aus einer Hand.

\section{LACKIERHAKEN}

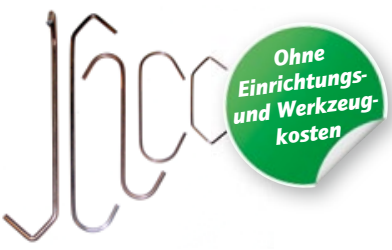

\section{LACKIERGESTELLE}

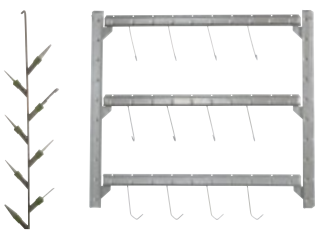

\section{MASKIERUNGEN}

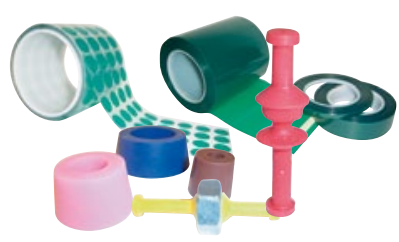

TRANSPORT- UND SAMMELGESTELLE
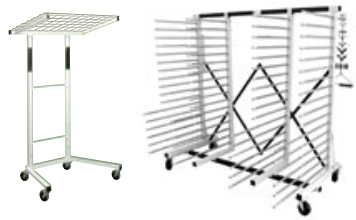

HILFSMITTEL

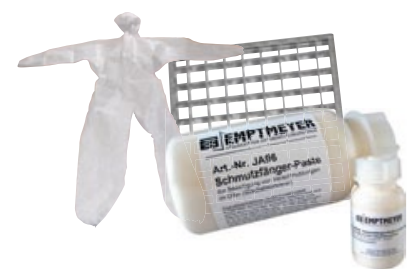

\section{SONDERARTIKEL}

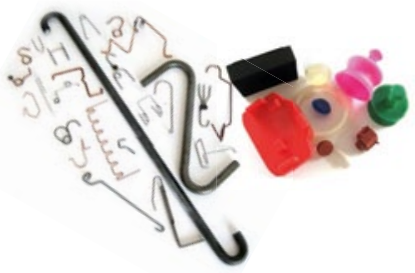

JÜRGEN EMPTMEYER

Senfdamm 28

49152 Bad Essen

+49(0) 5472/955 00-0

Online-Shop: www.emptmeyer.de 